\title{
Smartphone Screen Time Among University Students in Lebanon and Its Association With Insomnia, Bedtime Procrastination, and Body Mass Index During the COVID-19 Pandemic: A Cross-Sectional Study
}

\author{
Sajida Fawaz Hammoudi ${ }^{1 *}$, Hussein Walid Mreydem ${ }^{1 *}$, Bayan Tarek Abou Ali', \\ Nada Omar Saleh', Seockhoon Chung ${ }^{2 \otimes}$, Souheil Hallit ${ }^{3,6}$, and Pascale Salameh ${ }^{4,5,6} \bowtie$ \\ ${ }^{1}$ Faculty of Medical Sciences, Lebanese University, Beirut, Lebanon \\ ${ }^{2}$ Department of Psychiatry, Asan Medical Center, University of Ulsan College of Medicine, Seoul, Republic of Korea \\ ${ }^{3}$ Faculty of Medicine and Medical Sciences, Holy Spirit University of Kaslik (USEK), Jounieh, Lebanon \\ ${ }^{4}$ Department of Basic Sciences at the Faculty of Medical Sciences, Lebanese University, Beirut, Lebanon \\ ${ }^{5}$ Department of Primary Care and Population Health, University of Nicosia Medical School, Nicosia, Cyprus \\ ${ }^{6}$ Institut National de Santé Publique, Epidémiologie Clinique et Toxicologie-Liban (INSPECT-LB), Beirut, Lebanon
}

\begin{abstract}
Objective We aimed to investigate the association of increased smartphone screen time with insomnia, bedtime procrastination, depression, anxiety, body mass index (BMI), and physical activity during the coronavirus disease of 2019 (COVID-19) pandemic.

Methods An online survey was performed for university students from all regions of Lebanon during the lockdown. The survey included questionnaires about smartphone screen time, diet, physical activity, psychological symptoms, and bedtime procrastination. We defined 6 hours of smartphone use as critical based on a survey done in United States.

Results Among female students, smartphone use duration, physical activity levels, BMI, depression, anxiety, and insomnia severity were significantly higher than in male. When we stratified participants based on 6 hours of smartphone use, females, unhealthy food consumption, insomnia, anxiety, depression, and bedtime procrastination were significantly higher in the group with $\geq 6$ hours of smartphone use. When we divided based on 7 hours, physical activity and body weight also differed between the two groups. Logistic regression analysis revealed that female, overweight, insomnia, and bedtime procrastination were significant predictors of a phone screen time of 7 hours.
\end{abstract}

Conclusion Our findings suggest that adults should be more cautious and responsible when using smartphones and be more concerned about the health-related risks.

Psychiatry Investig 2021;18(9):871-878

Keywords COVID-19; Screen time; BMI; Insomnia; Bedtime procrastination.

\section{INTRODUCTION}

The coronavirus disease of 2019 (COVID-19) pandemic continues to spread worldwide and claim lives, despite the start of vaccination drives. The number of cases at the beginning of 2021 was 5 million, which decreased to 2.5 million by mid-Jan- uary; however, these numbers have started gradually increasing again. The American and European continents account for more than $80 \%$ of cases and deaths. The number of cases and deaths continue to increase in various parts of the world, except in Africa. ${ }^{1}$

From January 3, 2020, to March 17, 2021, there were 423,433

\footnotetext{
Received: April 8, 2021 Revised: June 15, 2021 Accepted: July 21, 2021

$\triangle$ Correspondence: Seockhoon Chung, MD, PhD

Department of Psychiatry, Asan Medical Center, University of Ulsan College of Medicine, 86 Olympic-ro 43-gil, Songpa-gu, Seoul 05505, Republic of Korea Tel: +82-2-3010-3411, Fax: +82-2-485-8381, E-mail: schung@amc.seoul.kr

$\square$ Correspondence: Pascale Salameh, PharmD, MPH, PhD, HDR

Department of Primary Care and Population Health, University of Nicosia Medical School, 93 Agiou Nikolaou Street, Engomi 2408 Nicosia, Cyprus

Tel: +961-3-385542, E-mail: pascalesalameh1@hotmail.com

*These authors contributed equally to this work.

(a) This is an Open Access article distributed under the terms of the Creative Commons Attribution Non-Commercial License (https://creativecommons.org/licenses/by-nc/4.0) which permits unrestricted non-commercial use, distribution, and reproduction in any medium, provided the original work is properly cited.
} 
confirmed cases of COVID-19 and 5,474 deaths in Lebanon, according to the World Health Organization (WHO). As of March 14, 2021, a total of 95,888 vaccine doses have been administered. ${ }^{2}$ On February 8, 2021, the Lebanese government announced restrictions on movement and public services to curb the spread of COVID-19 in the country. During the third phase of the lifting of lockdown restrictions, permissions to go out to markets, banks, and other crowded places were still required to be taken via the online platform. Moreover, the Lebanese government set limited operating hours for each of these aforementioned sectors. ${ }^{3}$ Regarding schools and universities, classes and exams are still being held online.

The COVID-19 pandemic led to a significant shift from traditional learning to e-learning, which tremendously transformed the learning process. This shift to online services not only occurred in the education sector but in all aspects of life. People worldwide, whether they were students, workers, or even unemployed, started relying on digital screens to learn, perform tasks, and communicate with others. Mobile phones, iPads, personal computers (PCs), and tablets have become omnipresent in our everyday life, and their use has dramatically catapulted. With each lockdown, the use of digital screens has soared, giving rise to drastic health implications. A study among 1,033 participants conducted in China reported that $70 \%$ of the participants spent more time looking at screens after the start of the COVID-19 pandemic. ${ }^{4}$ Furthermore, a study conducted in nine European countries found that approximately $65 \%$ of the 4,108 participants reported increased screen time during the pandemic. $^{5}$

There is evidence that increased screen time is associated with sleep disorders, depression, anxiety, obesity, and other noncommunicable diseases. ${ }^{6}$ Previous studies have shown that increasing levels of screen time were generally linked to progressively lower psychological well-being. In terms of relative risk, users with longer screen times were associated with twice the risk of low well-being compared to those with shorter screen times. Furthermore, users with longer screen times were also significantly more likely to have been diagnosed with anxiety or depression. ${ }^{7}$ Furthermore, in a cross-sectional study among 9,846 adolescents, $90 \%$ of the participants reported using digital devices within the last hour before lights-out, which was associated with prolonged sleep onset latency. ${ }^{8}$ In addition to insomnia, recent studies have investigated bedtime procrastination, which is defined as "going to bed later than intended despite absence of external reasons." In addition to the effects on mental health, many observational studies found relationships between screen media exposure and increased risk of obesity, most probably due to exposure to high-calorie, lownutrient foods and beverages. ${ }^{10}$

The decision taken by the Lebanese government to imple- ment a complete lockdown in January 2021, due to the massive increase in the number of COVID-19 cases, gave us the ideal opportunity to study the associations between increased use of electronic screens and various health aspects. Previous studies have suggested an association between increased screen time and various health problems; ${ }^{6}$ however, none of them investigated various mental health problems and insomnia. In addition, neither of the studies was conducted in a developing country such as Lebanon. In this study, we aimed to investigate whether increased duration of phone use was associated with sleep, bedtime procrastination, depression, anxiety, unhealthy eating habits, and physical inactivity among university students in Lebanon.

\section{METHODS}

\section{Participants and procedures}

Convenience sampling was used as the questionnaire was posted online using Google forms, and the link was shared via snowball technique. The responses were collected from 591 University Students across Lebanon and the survey was distributed from February 7, 2021, to February 14, 2021. Participation in the survey was completely voluntary. The study protocol was approved by the Institutional Review Board of Hôspital Psychiatrique de la Croix (IRB acceptance number: HPC-002-2021). Sociodemographic data on sex, age, weight, height, and region of residence were collected in the first part of the questionnaire. The questionnaire used was in common with another study done on school students.

\section{Assessments}

\section{Measuring screen time}

Questions that directly asked about screen time usage were used to quantify the number of hours spent by each participant on the phone.

\section{Measuring physical activity, body mass index, and \\ dietary intake}

Body mass index (BMI) was calculated by collecting each participant's weight in kilograms and height in meters. Physical activity was assessed through the question "How do you describe your average physical activity?", and the students could respond with "Light (walking slowly [i.e., shopping, walking around the office], sitting at your computer, making the bed, eating, preparing food, and washing dishes)," "Moderate (sweeping the floor, walking briskly, slow dancing, vacuuming, washing windows, or playing basketball)," or "Vigorous (running $>5$ miles per hour, swimming, shoveling, soccer, jumping rope, or carrying heavy loads [e.g., bricks])." 
Regarding food habits, participants were asked to classify dietary intake during the month of the survey as "healthy food (vegetables, fruits, beans, chicken, or fish)," "sometimes healthy food," or "unhealthy food (fast food, fries, or sweets)."

\section{Patient Health Questionnaire-9 items}

The Patient Health Questionnaire-9 (PHQ-9) was used to measure depression severity among the participants. The scale consists of nine questions, and each question has a possible score ranging from 0 (not at all) to 3 (nearly every day). An overall score, out of a total of 27 points, was then calculated for each participant, and depression severity was classified as follows: $0-4$, no depression; 5-9, mild depression; $10-14$, moderate depression; 15-19, moderately severe depression; and 20-27, severe depression. We used the Arabic version of the PHQ-9 scale and defined clinical depression as a PHQ-9 score of $\geq 10$. $^{11,12}$

\section{Generalized Anxiety Disorder-7 items}

The Generalized Anxiety Disorder-7 (GAD-7) scale was used to assess anxiety levels among the participants. It is a 7-item questionnaire with four possible choices for each question, with scores ranging from 0 (never) to 3 (nearly every day). The total possible score is 21 . Scores of 5, 10, and 15 are cut-off points for mild, moderate, and severe anxiety, respectively. We used the Arabic version of the GAD-7 scale and defined clinical anxiety as a GAD-7 score of $\geq 10$. $^{12,13}$

\section{Insomnia Severity Index}

To study insomnia levels among the participants, the Insomnia Severity Index (ISI) was used, which consists of seven questions. The possible score for each question ranges from 0 (none) to 4 (very severe). A total score out of 28 points was then calculated for each participant. Participants' insomnia levels were classified as follows: $0-7$, no clinically significant insomnia; 8-14, sub-threshold insomnia; 15-21, clinical insomnia (moderate severity); and 22-28, clinical insomnia (severe). We used the Arabic version of the ISI and defined clinical insomnia as an ISI score of $\geq 8 .{ }^{14,15}$

\section{Bedtime procrastination}

Bedtime procrastination was estimated using the Bedtime Procrastination Scale that consists of nine questions, with possible scores ranging from 1 (almost never) to 5 (almost always). Questions 2, 3, 7, and 9 were inversely scored, as indicated in the scale's manual. The sum of the scores was calculated, and participants with the highest scores were considered to show the greatest bedtime procrastination. ${ }^{16}$ The Arabic version of the scale was not formally published; thus, we translated this scale to Arabic with the permission of the original developers.
A Cronbach's alpha $=0.75$ was observed in a sample of this study.

\section{Statistical analysis}

Continuous variables and categorical variables were analyzed using the Mann-Whitney U test and chi-square test, respectively, for between-group analyses. Correlation analysis of the PHQ-9 and GAD-7 scores was conducted using Spearman correlation coefficients among the variables due to the lack of a normal distribution. Logistic regression analysis was performed to examine the predictors of phone use duration. Demographic characteristics and clinical data were summarized as means $\underline{ \pm}$ standard deviations. The significance level for all analyses was defined as a two-tailed $p$-value of $<0.05$. SPSS version 21.0 for Windows (IBM Corp., Armonk, NY, USA) was used for statistical analyses.

\section{RESULTS}

A total of 591 university students in Lebanon participated in this study. They were from Beirut $(\mathrm{n}=61,10.3 \%)$, Mount Lebanon $(\mathrm{n}=86,14.6 \%)$, the north region $(\mathrm{n}=56,9.5 \%)$, Akkar $(\mathrm{n}=5,0.8 \%)$, the south region $(\mathrm{n}=60,10.2 \%)$, Nabatieh $(\mathrm{n}=31$, $5.2 \%)$, Bekaa $(n=246,41.6 \%)$, and Baalbak-Hermel $(n=46$, $7.8 \%)$. Their majors were medicine $(\mathrm{n}=60,10.2 \%)$, dentistry $(n=4,0.7 \%)$, pharmacy $(n=30,5.1 \%)$, sciences $(n=97,16.4 \%)$, engineering $(n=50,8.5 \%)$, architecture $(n=9,1.5 \%)$, languages $(n=56,9.5 \%)$, and others $(n=283,47.9 \%)$. Among the participants, 111 (18.8\%) were male and 480 (81.2\%) were female. Between male and female students, there was a significant difference in phone use duration $(\mathrm{p}<0.001)$, physical activity level $(\mathrm{p}<0.001)$, and BMI $(\mathrm{p}=0.035)$ (Table 1$)$. The severity of depression (PHQ-9 scores), general anxiety (GAD-7 scores), and insomnia (ISI scores) was significantly higher among female students than among male students. There was no significant difference in bedtime procrastination between the two groups.

Regarding phone screen times among the university students, the prevalence of insomnia, anxiety, depression, and overweight increased with increasing phone screen time (Figure 1).

When we stratified the participants into two groups using a phone screen time of 6 hours as the cut-off value based on the survey of screen time trends during the pandemic in the United States, ${ }^{17}$ the proportion of female students; proportion of students with unhealthy food consumption; and levels of insomnia, anxiety, depression, and bedtime procrastination were higher in the group with a screen time of $\geq 6$ hours than in those with a screen time of $<6$ hours (Table 2). When we divided the participants into two groups using a phone screen time of 7 hours as the cut-off value, about one-third of the students were classified into the group with a screen time of $\geq 7$ hours ( $n=192$, $32.5 \%$ ). The proportion of female students; proportion of stu- 
Table 1. Demographic characteristics of the respondents $(\mathrm{N}=591)$

\begin{tabular}{|c|c|c|c|}
\hline Variables & Male $(\mathrm{N}=111)$ & Female $(\mathrm{N}=480)$ & p-value \\
\hline Age, years & $21.3 \pm 5.1$ & $21.1 \pm 3.8$ & 0.797 \\
\hline \multicolumn{3}{|c|}{ How much time per day do you spend using your phone during this pandemic? } & $<0.001^{*}$ \\
\hline Never & $1(0.9)$ & $2(0.4)$ & \\
\hline Less than 1 hour & $1(0.9)$ & $6(1.3)$ & \\
\hline $1-2$ hours & $14(12.6)$ & $24(5.0)$ & \\
\hline 3 hours & $27(24.3)$ & $57(11.9)$ & \\
\hline $4-5$ hours & $25(22.5)$ & $128(26.7)$ & \\
\hline 6-7 hours & $21(18.9)$ & $93(19.4)$ & \\
\hline$>7$ hours & $22(19.8)$ & $170(35.4)$ & \\
\hline \multicolumn{3}{|l|}{ How do you describe your average physical activity? } & $<0.001^{*}$ \\
\hline Light & $55(49.5)$ & $259(54.0)$ & \\
\hline Moderate & $37(33.3)$ & $211(44.0)$ & \\
\hline Vigorous & $19(17.1)$ & $10(2.1)$ & \\
\hline \multicolumn{3}{|l|}{ During this month, your food mainly constituted } & 0.334 \\
\hline Healthy food & $37(33.3)$ & $129(26.9)$ & \\
\hline Sometimes healthy food & $53(47.7)$ & $263(54.8)$ & \\
\hline Unhealthy food & $21(18.9)$ & $88(18.3)$ & \\
\hline Body mass index & $23.9 \pm 3.8$ & $23.0 \pm 4.1$ & $0.035^{*}$ \\
\hline Overweight (BMI $\geq 25)$ & $34(30.6)$ & $115(24.1)$ & 0.182 \\
\hline \multicolumn{4}{|l|}{ Rating scales } \\
\hline Patient Health Questionnaire-9 (PHQ-9) & $10.3 \pm 6.3$ & $12.3 \pm 6.3$ & $0.004^{*}$ \\
\hline Clinical depression (PHQ-9 score $\geq 10$ ) & $57(51.4)$ & $290(60.4)$ & 0.087 \\
\hline Generalized Anxiety Disorder-7 (GAD-7) & $8.1 \pm 5.8$ & $10.8 \pm 5.8$ & $<0.001^{*}$ \\
\hline Clinical anxiety (GAD-7 score $\geq 10$ ) & $40(36.0)$ & $263(54.8)$ & $<0.001^{*}$ \\
\hline Insomnia Severity Index (ISI) & $12.6 \pm 6.9$ & $14.5 \pm 6.8$ & $0.007^{*}$ \\
\hline Clinical insomnia (ISI score $\geq 8$ ) & $83(74.8)$ & $396(82.5)$ & $0.044^{*}$ \\
\hline Bedtime procrastination & $30.2 \pm 6.7$ & $30.9 \pm 7.0$ & 0.780 \\
\hline
\end{tabular}

Data are presented as mean \pm standard deviation or $\mathrm{N}(\%)$. *statistically significant. SD, standard deviation; BMI, body mass index

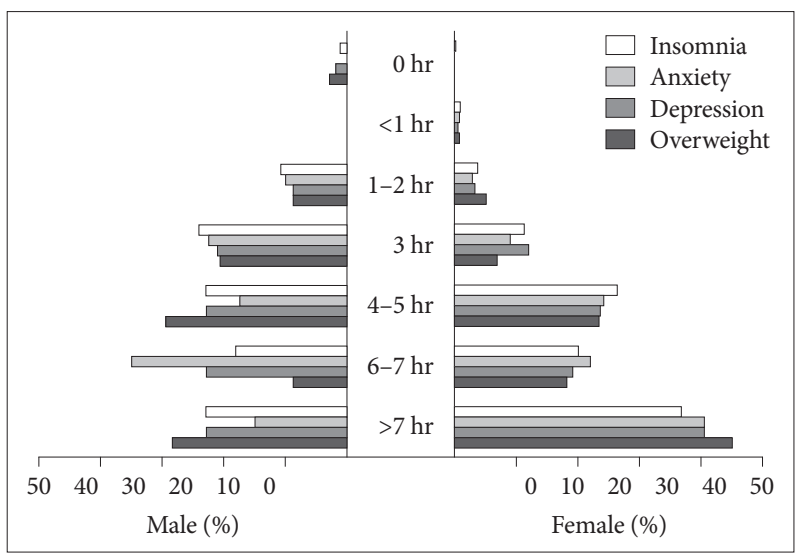

Figure 1. Prevalence of clinical insomnia, anxiety, depression, and overweight among the study participants. This figure presents the proportions of male and female students with clinical insomnia, anxiety, depression, and overweight stratified by the duration of screen time. dents who reported unhealthy food consumption and physical inactivity; and levels of BMI, insomnia, anxiety, depression, and bedtime procrastination were higher, whereas the proportion of students who reported vigorous physical activity was lower in the group with a screen time of $\geq 7$ hours than in those with a screen time of $<7$ hours.

In this study, we defined 7 hours of phone use as critical screen time. Logistic regression analysis revealed that female sex (adjusted odds ratio $[\mathrm{aOR}]=2.19,95 \%$ confidence interval $[\mathrm{CI}]=$ 1.27-3.77), overweight status ( $\mathrm{aOR}=1.85,95 \% \mathrm{CI}=1.22-1.28)$, insomnia $(\mathrm{aOR}=1.06,95 \% \mathrm{CI}=1.02-1.10)$, and bedtime procrastination $(\mathrm{aOR}=1.03,95 \% \mathrm{CI}=1.00-1.07)$ were significant predictors of a phone screen time of $\geq 7$ hours (Table 3 ).

\section{DISCUSSION}

The COVID-19 pandemic has not only affected the physical 
Table 2. Differences in clinical variables according to phone use duration

\begin{tabular}{|c|c|c|c|c|c|c|}
\hline Variables & $<6$ hours $(\mathrm{N}=285)$ & $\geq 6$ hours $(\mathrm{N}=306)$ & p-value & $<7$ hours $(\mathrm{N}=399)$ & $\geq 7$ hours $(\mathrm{N}=192)$ & $\mathrm{p}$-value \\
\hline Age, years & $21.5 \pm 4.3$ & $20.9 \pm 3.8$ & 0.103 & $21.3 \pm 4.0$ & $20.8 \pm 4.2$ & 0.180 \\
\hline Sex (female) & $217(76.1)$ & $263(85.9)$ & $0.003^{*}$ & $310(77.7)$ & $170(88.5)$ & $0.002^{*}$ \\
\hline \multicolumn{3}{|c|}{ How do you describe your average physical activity? } & 0.690 & & & $0.015^{*}$ \\
\hline Light & $148(51.9)$ & $168(54.2)$ & & $197(49.4)$ & $117(60.9)$ & \\
\hline Moderate & $121(42.5)$ & $127(41.5)$ & & $178(44.6)$ & $70(36.5)$ & \\
\hline Vigorous & $16(5.6)$ & $13(4.2)$ & & $24(6.0)$ & $5(2.6)$ & \\
\hline \multicolumn{3}{|c|}{ During this month, your food mainly constituted } & $0.003^{*}$ & & & $0.007^{*}$ \\
\hline Healthy food & $92(32.3)$ & $74(24.2)$ & & $123(30.8)$ & $43(22.4)$ & \\
\hline Sometimes healthy food & $155(54.4)$ & $161(52.6)$ & & $215(53.9)$ & $101(52.6)$ & \\
\hline Unhealthy food & $38(13.3)$ & $71(23.2)$ & & $61(15.3)$ & $48(25.0)$ & \\
\hline Overweight $(\mathrm{BMI} \geq 25)$ & $63(22.3)$ & $86(28.1)$ & 0.108 & 87 (21.9) & $62(32.3)$ & $0.008^{*}$ \\
\hline \multicolumn{7}{|l|}{ Rating scales } \\
\hline Patient Health Questionnaire-9 & $10.5 \pm 5.8$ & $13.2 \pm 6.6$ & $<0.001^{*}$ & $11.2 \pm 6.1$ & $13.4 \pm 6.7$ & $<0.001^{*}$ \\
\hline Generalized Anxiety Disorder-7 & $9.1 \pm 5.4$ & $11.4 \pm 6.6$ & $<0.001^{*}$ & $9.8 \pm 5.9$ & $11.3 \pm 6.0$ & $0.003^{*}$ \\
\hline Insomnia Severity Index & $12.3 \pm 6.2$ & $15.9 \pm 7.0$ & $<0.001^{*}$ & $13.0 \pm 6.4$ & $16.5 \pm 7.2$ & $<0.001^{*}$ \\
\hline Bedtime procrastination & $30.2 \pm 6.7$ & $32.3 \pm 6.5$ & $<0.001^{*}$ & $30.4 \pm 6.5$ & $33.1 \pm 6.7$ & $<0.001^{*}$ \\
\hline
\end{tabular}

Data are presented as mean \pm standard deviation or $\mathrm{N}(\%)$. *statistically significant. SD, standard deviation; BMI, body mass index

Table 3. Results of the logistic regression analysis exploring predicting variables for phone use $\geq 7$ hours

\begin{tabular}{|c|c|c|c|c|c|c|}
\hline Variables & cOR & $95 \% \mathrm{CI}$ & $\mathrm{p}$-value & $\mathrm{aOR}$ & $95 \% \mathrm{CI}$ & $\mathrm{p}$-value \\
\hline Sex (female) & 2.22 & $1.34-3.69$ & $0.002^{*}$ & 2.19 & $1.27-3.77$ & $0.005^{*}$ \\
\hline Age & 0.97 & $0.92-1.02$ & 0.182 & 0.96 & $0.91-1.01$ & 0.126 \\
\hline Unhealthy food & 1.85 & $1.21-2.83$ & $0.005^{*}$ & 1.41 & $0.89-2.25$ & 0.140 \\
\hline Vigorous activity & 0.65 & $0.40-1.06$ & 0.079 & 0.64 & $0.22-1.82$ & 0.400 \\
\hline Overweight (BMI $\geq 25 \mathrm{~kg} / \mathrm{m}^{2}$ ) & 1.70 & $1.16-2.50$ & $0.007^{*}$ & 1.85 & $1.22-2.81$ & $0.004^{*}$ \\
\hline \multicolumn{7}{|l|}{ Rating scale scores } \\
\hline PHQ-9 & 1.06 & $1.03-1.09$ & $<0.001^{*}$ & 1.004 & $0.96-1.06$ & 0.872 \\
\hline GAD-7 & 1.05 & $1.01-1.08$ & $0.004^{*}$ & 0.98 & $0.93-1.03$ & 0.431 \\
\hline ISI & 1.08 & $1.05-1.11$ & $<0.001^{*}$ & 1.06 & $1.02-1.10$ & $0.002^{*}$ \\
\hline Bedtime procrastination & 1.07 & $1.04-1.10$ & $<0.001^{*}$ & 1.03 & $1.00-1.07$ & $0.049^{*}$ \\
\hline
\end{tabular}

Table 3 shows the associations of phone use more than 7 hours with the outcome variables among the university students surveyed. *statistically significant. cOR, crude odds ratio; aOR, adjusted odds ratio; CI, confidence interval; BMI, body mass index; PHQ-9, Patient Health Questionnaire-9; GAD-7, Generalized Anxiety Disorder-7; ISI, Insomnia Severity Index

health of individuals but has also greatly impacted mental health and human behaviors. In the present study conducted in a large sample of university students in Lebanon, we investigated the relationship between increased screen time-especially smartphone usage, which was shown in another study from India to be the primary device used by university students ${ }^{18}$ - and different health aspects and behaviors, including physical activity, eating habits, depression severity, anxiety levels, insomnia, and bedtime procrastination during the pandemic. Additionally, we aimed to find an estimate of screen time above which poor lifestyle habits and health outcomes become significant. Among the 591 participants, 111 were male and 480 were fe- male, and there was no significant difference in the age between the groups ( $p=0.80$ ). We found a significant difference in phone use time, physical activity level, and BMI between male and female students.

This study found that as the screen time increased, the proportion of participants with insomnia, anxiety, depression, and $\mathrm{BMI} \geq 25 \mathrm{~kg} / \mathrm{m}^{2}$ (overweight) also increased. These findings were comparable with those in the aforementioned study from India, which found that increased cell phone use at night was negatively correlated with sleep duration; the authors attributed this to blue light exposure, which suppresses melatonin production necessary for sleep and in turn, leads to insufficient 
sleep. ${ }^{18}$ Additionally, this study showed that increased screen time was associated with higher depressive symptomatology. ${ }^{19}$ Regarding anxiety assessment, our results were consistent with those of another study, which showed that increased use of screens was associated with an increase in anxiety symptoms among participants. ${ }^{20}$ In another study conducted in Poland, it was shown that the pandemic led to decreased physical activity and exercise, even though home-based training programs appeared in the media. This was due to the inability of people to adapt to training at home. This led to weight gain among people during lockdown, which resulted in an increased BMI. ${ }^{21}$ Hence, the results of our study conducted in Lebanon were consistent with those of other studies from different regions worldwide and systematic reviews published during the pandemic.

Before the pandemic, a study conducted in Turkey found that the majority of university students used their phones mostly at night and/or in the evening and for approximately 5 hours per day, and a positive correlation was found between Smartphone Addiction Scale scores and sleep quality scores. The authors suggested that the sleep problems might have been due to the direct effect of depression and anxiety on sleep, as depression and anxiety predicted poor sleep quality.22 Another study reported that bright light suppresses the nocturnal changes in melatonin concentration and other physiological indicators of the human circadian rhythm. ${ }^{23}$ Regarding bedtime procrastination, a study conducted in the Republic of Korea found that individuals who spent more time on their phones over a duration of 24 hours and used their phone 3 hours prior to bedtime were in the high bedtime procrastination group. ${ }^{9}$ Hence, the results of these studies conducted before the COVID-19 pandemic were consistent with our results.

February 2021, the month during which the survey was rolled out, was critical for the entire world as this was the start of vaccine administration; it was also a critical month for Lebanon since the country was awaiting a decision regarding the vaccination trial. The Lebanese population was anticipating the arrival of the vaccines, which became available in the country on February 13, 2021. During the time of questionnaire distribution, vaccination drives had not yet started in Lebanon, and the high numbers of COVID-19 cases and death were worrisome. The total lockdown in Lebanon did little to slow down the rise in daily cases. Additionally, during this time, Lebanon was still facing an economic crisis, which greatly affected the standard of living of the population. All these factors had adverse effects on Lebanese citizens and university students.

In this study, food consumption and physical activity patterns were different between groups based on phone use duration. Food consumption patterns among students in Lebanese universities were reported in a previous study that included 3,348 students. ${ }^{24}$ It was shown that more than a quarter of the participants adopted a vegetarian/low-calorie dietary pattern, characterized by high consumption of plant-based foods and lower consumption of white bread and Western foods. A mixed dietary pattern was adopted by slightly more than one-third of the university students. The Westernized dietary pattern was characterized by the consumption of fast foods, beverages, and desserts, and this was adopted by the largest proportion of university students. ${ }^{24}$ Regarding physical activity, a study conducted in Lebanon before the pandemic found that the prevalence of physical activity was high among students. In addition, it was shown that the physical activity level was a determinant of weight status and percentage of body fat. Previously, it was reported that 5 or more hours of daily phone use was associated with an increased risk of obesity, ${ }^{25}$ and these results were consistent with those of our study.

Sex-specific differences may also exist when it comes to physical activity. Female students were found to be less involved in vigorous physical activity than male students. ${ }^{26}$ In addition, the prevalence of clinical depression, clinical anxiety, and clinical insomnia was higher among female students than among male students; $60.4 \%, 54.8 \%$, and $82.5 \%$ of female students had clinical depression, clinical anxiety, and clinical insomnia, respectively, whereas the corresponding proportions among male students were $51.4 \%, 36.0 \%$, and $74.8 \%$, respectively. However, there were no differences in bedtime procrastination. The results of depression and anxiety were consistent with those of a systematic review that showed that men and women had different coping mechanisms to deal with these conditions, i.e., women tended to internalize their feelings more than men. Hence, more screen time among women indicates that they would have less time to express their feelings, while men tend to shift their attention toward other affairs. This may explain the results of our study, which showed that the female sex was more likely to be associated with different study outcomes. ${ }^{19}$

In this study, regarding the estimation of phone screen time and its association with different aspects, the results were divided using 6 hours and 7 hours of phone screen time as cutoff values, which led to the identification of 7 hours of phone use as the critical screen time. At first, we hypothesized that 6 hours of phone use may be critical for University students based on the survey done in United States which reported that the smartphone use increased up to 5.67 hours per day during pandemic. ${ }^{17}$ We compared participants with screen times of $\geq 6$ hours or $\geq 7$ hours to those with screen times of $<6$ hours or $<7$ hours, respectively, and it was shown that the former groups had a higher proportion of female students; higher proportion of students with unhealthy food consumption; and higher levels of insomnia, anxiety, depression, and bedtime procrastination than the latter groups. For adults, there is no proposed restriction for phone screen time as they have autonomy over 
their phone use. However, we observed that increased phone use duration was associated with higher levels of insomnia, depression, and anxiety, as well as a BMI of $\geq 25 \mathrm{~kg} / \mathrm{m}^{2}$ (overweight). We propose 7 hours of phone use as the critical screen time for adults, in accordance with the higher prevalence of mental or physical health problems, although it would be ideal to decrease screen time as much as possible.

The higher prevalence of depression (male, $51.4 \%$; female, $60.4 \%$ ), anxiety (male, $36.0 \%$; female, $54.8 \%$ ), and insomnia (male, $74.8 \%$; female, $82.5 \%$ ) may be a limitation of this study. The COVID-19 pandemic might have increased the risk of psychological stress or psychiatric symptoms. This study was also limited by its cross-sectional design, which precludes the investigation of causal relationships. The data were collected only 6 months after the Beirut explosion, which was one of the biggest explosions in history. In addition, our study was conducted during the second wave of the COVID-19 pandemic, when hospitals were at full capacity and the number of deaths had significantly increased. During that time, the Lebanese population was not only experiencing health and security problems but also experiencing economic problems due to the drop in the value of the Lebanese pound to record levels. As this study was conducted during one of the most difficult periods Lebanon has ever experienced, the associations of increased screen time with depression, anxiety, insomnia, and bedtime procrastination could have been overestimated.

Collection of data using an online survey had its strengths and limitations. One strength of this type of collection was the ability to recruit a large sample from different regions of Lebanon even during complete lockdown, while preventing the risk of disease spread that could occur had we done face-toface surveys. Another strength of this study was the timing of distribution of the questionnaire (i.e., during a lockdown wherein people were quarantined), making this time optimal for assessing increased screen time. One limitation is that we could not assess the physical activity and diet in detail, and we considered that the physical activities or diet may be somewhat consistent during the lockdown. Another limitation of this study was that we only gathered information on smartphone use. Other electronic devices such as laptops, desktops, or tablet PCs, might influence the results of this study. Lastly, a limitation of using this kind of questionnaire was that the responses were self-reported, which could have led to objective bias.

In conclusion, there was a significant difference in phone use duration, physical activity, and BMI between male and female students in Lebanon. The severity of depression (PHQ-9 scores), general anxiety (GAD-7 scores), and insomnia (ISI scores) was significantly higher among female students than among male students. There was no significant difference in bedtime procrastination between the two groups. We defined 7 hours of screen time as the critical screen time for university students. The group with $\geq 7$ hours of screen time had a higher proportion of female students; higher proportion of students with unhealthy food consumption; lower proportion of students who reported vigorous physical activity; and higher levels of BMI, insomnia, anxiety, depression, and bedtime procrastination. This study may help pave the way for future research on determining a healthy screen time. This would help guide people to use screens for limited periods and to reduce the risk of several health problems.

\section{Availability of Data and Material}

The datasets generated or analyzed during the study are available from the corresponding author on reasonable request.

\section{Conflicts of Interest}

The authors have no potential conflicts of interest to disclose.

\section{Author Contributions}

Conceptualization: Pascale Salameh, Souheil Hallit, Sajida Fawaz Hammoudi, Hussein Walid Mreydem, Seockhoon Chung. Data curation: Sajida Fawaz Hammoudi, Hussein Walid Mreydem, Bayan Tarek Abou Ali, Nada Omar Saleh. Formal analysis: Seockhoon Chung. Funding acquisition: Pascale Salameh. Investigation: Seockhoon Chung. Methodology: Pascale Salameh, Souheil Hallit, Seockhoon Chung. Project administration: Hussein Walid Mreydem. Resources: Seockhoon Chung. Software: Seockhoon Chung. Supervision: Pascale Salameh, Seockhoon Chung. Validation: Seockhoon Chung. Visualization: Hussein Walid Mreydem, Sajida Fawaz Hammoudi. Writing_original draft: Sajida Fawaz Hammoudi, Hussein Walid Mreydem, Bayan Tarek Abou Ali, Nada Omar Saleh. Writing-review \& editing: Seockhoon Chung, Pascale Salameh, Souheil Hallit.

\section{ORCID iDs}

Sajida Fawaz Hammoudi Hussein Walid Mreydem Bayan Tarek Abou Ali Nada Omar Saleh Seockhoon Chung Souheil Hallit

Pascale Salameh https://orcid.org/0000-0002-6315-9209 https://orcid.org/0000-0002-0963-5139 https://orcid.org/0000-0002-8920-2957 https://orcid.org/0000-0002-2795-3448 https://orcid.org/0000-0002-9798-3642 https://orcid.org/0000-0001-6918-5689 https://orcid.org/0000-0002-4780-0772

\section{Funding Statement \\ None.}

\section{Acknowledgments}

We would like to appreciate all the Lebanon people who voluntarily participated in the survey.

\section{REFERENCES}

1. WHO. Weekly Epidemiological Update On COVID-19. Geneva: WHO; 2021.

2. WHO. Lebanon: WHO Coronavirus Disease (COVID-19) Dashboard. Geneva: WHO; 2021.

3. US Embassy. COVID-19 Information. Beirut: U.S. Embassy In Lebanon; 2021.

4. Hu Z, Lin X, Chiwanda Kaminga A, Xu H. Impact of the COVID-19 epidemic on lifestyle behaviors and their association with subjective well-being among the general population in Mainland China: crosssectional study. J Med Internet Res 2020;22:e21176.

5. Pisot S, Milovanovic I, Simunic B, Gentile A, Bosnar K, Prot F, et al. 
Maintaining everyday life praxis in the time of COVID-19 pandemic measures (ELP-COVID-19 survey). Eur J Public Health 2020;30:11811186.

6. Stiglic N, Viner RM. Effects of screentime on the health and well-being of children and adolescents: a systematic review of reviews. BMJ Open 2019;9:e23191.

7. Twenge JM, Campbell WK. Associations between screen time and lower psychological well-being among children and adolescents: evidence from a population-based study. Prev Med Rep 2018;12:271-283.

8. Hysing M, Pallesen S, Stormark KM, Jakobsen R, Lundervold AJ, Sivertsen B. Sleep and use of electronic devices in adolescence: results from a large population-based study. BMJ Open 2015;5:e006748.

9. Chung SJ, An H, Suh S. What do people do before going to bed? A study of bedtime procrastination using time use surveys. Sleep 2020; 43:zsz267.

10. Robinson TN, Banda JA, Hale L, Lu AS, Fleming-Milici F, Calvert SL, et al. Screen media exposure and obesity in children and adolescents. Pediatrics 2017;140:S97-S101.

11. Kroenke K, Spitzer RL, Williams JB. The PHQ-9: validity of a brief depression severity measure. J Gen Intern Med 2001;16:606-613.

12. Sawaya H, Atoui M, Hamadeh A, Zeinoun P, Nahas Z. Adaptation and initial validation of the Patient Health Questionnaire-9 (PHQ-9) and the Generalized Anxiety Disorder-7 Questionnaire (GAD-7) in an Arabic speaking Lebanese psychiatric outpatient sample. Psychiatry Res 2016;239:245-252.

13. Spitzer RL, Kroenke K, Williams JB, Lowe B. A brief measure for assessing generalized anxiety disorder: the GAD-7. Arch Intern Med 2006; 166:1092-1097.

14. Morin CM, Belleville G, Belanger L, Ivers H. The Insomnia Severity Index: psychometric indicators to detect insomnia cases and evaluate treatment response. Sleep 2011;34:601-608.

15. Suleiman KH, Yates BC. Translating the insomnia severity index into Arabic. J Nurs Scholarsh 2011;43:49-53.

16. Kroese FM, De Ridder DT, Evers C, Adriaanse MA. Bedtime procrasti- nation: introducing a new area of procrastination. Front Psychol 2014; 5:611.

17. Wilkinson D. Screen Time Trends in The Age of COVID-19. Miami Beach, FL: SimpleTexting. Available at: https://simpletexting.com/ screen-time-survey/. Accessed June 15, 2021.

18. Majumdar P, Biswas A, Sahu S. COVID-19 pandemic and lockdown: cause of sleep disruption, depression, somatic pain, and increased screen exposure of office workers and students of India. Chronobiol Int 2020; 37:1191-1200.

19. Wang X, Li Y, Fan H. The associations between screen time-based sedentary behavior and depression: a systematic review and meta-analysis. BMC Public Health 2019;19:1524.

20. Boers E, Afzali MH, Conrod P. Temporal associations of screen time and anxiety symptoms among adolescents. Can J Psychiatry 2020;65: 206-208.

21. Gornicka M, Drywien ME, Zielinska MA, Hamulka J. Dietary and lifestyle changes during COVID-19 and the subsequent lockdowns among Polish adults: a cross-sectional online survey PLifeCOVID-19 study. Nutrients 2020;12:2324.

22. Demirci K, Akgonul M, Akpinar A. Relationship of smartphone use severity with sleep quality, depression, and anxiety in university students. J Behav Addict 2015;4:85-92.

23. Higuchi S, Motohashi Y, Liu Y, Ahara M, Kaneko Y. Effects of VDT tasks with a bright display at night on melatonin, core temperature, heart rate, and sleepiness. J Appl Physiol (1985) 2003;94:1773-1776.

24. Salameh P, Jomaa L, Issa C, Farhat G, Salame J, Zeidan N, et al. Assessment of dietary intake patterns and their correlates among university students in Lebanon. Front Public Health 2014;2:185.

25. Five or more hours of smartphone usage per day may increase obesity. Rockville, MD: ScienceDaily. Available at: https://www.sciencedaily. com/releases/2019/07/190725150918.htm. Accessed June 15, 2021.

26. Yahia N, Abdallah A, Achkar A, Rizk S. Physical activity and smoking habits in relation to weight status among Lebanese university students. Int J Health Res 2010;3:21-29. 